\title{
Ensemble-Empirical-Mode-Decomposition Based micro-Doppler signal separation and classification
}

\section{Huajie Chen*, Ping Lin}

Key Laboratory of Fundamental Science for National Defense-Communication Information Transmission and Fusion Technology,

Hangzhou Dianzi University,

Hangzhou, 310018, China

Emails: chj247@hdu.edu.cn; $\underline{664938946 @ q q . c o m}$

*Corresponding author

Khemraj Emrith, Pritesh Narayan, Yufeng Yao

Department of Engineering Design and Mathematics

University of the West of England

Coldharbour Lane, Frenchay

Bristol BS16 1QY

Emails: Khemraj.Emrith@uwe.ac.uk; Pritesh.Narayan@uwe.ac.uk; Yufeng.Yao@uwe.ac.uk

\begin{abstract}
The target echo signals obtained by Synthetic Aperture Radar (SAR) and Ground Moving Target Indicator (GMTI) platforms are mainly composed of two parts, the microDoppler signal and the target body part signal. The wheeled vehicle and the track vehicle are classified according to the different character of their micro-Doppler signal. In order to overcome the mode mixing problem in Empirical Mode Decomposition (EMD), Ensemble Empirical Mode Decomposition (EEMD) is employed to decompose the original signal into a number of Intrinsic Mode Functions (IMF). The correlation analysis is then carried out to select IMFs which have a relatively high correlation with the micro-Doppler signal. Thereafter, four discriminative features are extracted and Support Vector Machine (SVM) classifier is applied for classification. The experimental results show that the features extracted after EEMD decomposition are effective, with up $90 \%$ success rate for classification using one feature. In addition, these four features are complementary in different target velocity and azimuth angles.
\end{abstract}

Keywords: micro-Doppler; micro-motion; EEMD; IMF; wheeled/tracked vehicle; SAR/GMTI; signal separation; feature abstraction; vehicle classification; SVM

Reference to this paper should be made as follows: Chen, H., Lin, P., Emrith,K., Narayan, P. and Yao, Y. (xxxx) 'Ensemble-Empirical-Mode-Decomposition Based micro-Doppler signal separation and classification.', Int. J. Computer Applications in Technology, Vol. X, No. Y, pp.000-000.

Biographical notes: Huajie Chen was born in 1978. He received the B.S. degree in electrical engineering in 2001 and the Ph.D. degree in control theory and control engineering in 2006, from Zhejiang University, China. Since 2006, he has been with the School of Automation, Hangzhou Dianzi University, China. From 2008 to 2015, he was an Associate Professor in Pattern Recognition and Intelligence System at Hangzhou Dianzi University, China. From 2016, he has been a Professor in Pattern Recognition and Intelligence System at Hangzhou Dianzi University, China. His research interests include signal processing, pattern recognition, image processing and machine learning.

Ping Lin was born in 1992. She received the B.E. degree in Automation in 2014. Since 2014 she is studying for M.E. degree in control engineering degree at the School of Automation, Hangzhou Dianzi University, China. Her research interests include signal processing and image processing.

Khemraj Emrith is currently a Senior Lecturer in Engineering at the Bristol Robotics Lab (BRL), University of the West of England, UK. His work at the BRL is focused on the real-time modelling of dynamic 3D facial expressions and 3D surface texture modelling. He obtained his $\mathrm{PhD}$ (2008) from the Texture Lab, Heriot Watt University, Edinburgh, researching perceptual models for 3D surface texture retrieval. He also holds an MSc (MRes) in Computing and a BEng (Hons) in Computer Science and Engineering. His research interests include texture perception 
and classification, statistical learning for vision based systems, dimensionality reduction techniques, and higher-order statistics.

Pritesh Narayan is a Senior Lecturer in Aerospace Engineering and member of the Engineering, Modelling and Simulation research Group (EMSG) at the University of the West of England (Bristol, United Kingdom). He obtained his PhD in Unmanned Aerial Vehicle (UAV) trajectory planning from the Queensland University of Technology (Brisbane, Australia). Pritesh's research interests include Autonomous UAV planning and decision making, and Aircraft Guidance, Navigation and Control.

Yufeng Yao is Professor and Head of Engineering Modelling and Simulation group at University of the West of England (UWE) Bristol, UK. He received PhD from Glasgow University, Scotland and then was working as Research Fellow at several UK Universities, including Queen Marry College, London, and University of Southampton before joining Siemens Industrial Turbo-Machinery Ltd as Senior Aerothermodynamics Engineer. He returned to academics as Senior Lecturer and promoted to Reader at Kingston University London. He recently joined UWE as a full Professor. His research interest includes engineering flow and heat transfer modelling and simulation, large-scale data analysis, verification and validation.

\section{Introduction}

The need to gain better and highly accurate battlefield awareness has boosted a number of technological advancements in the field of military surveillance. Recent evolution in battlefield operations has thus seen the combined use of Synthetic Aperture Radar (SAR) and Ground Moving Target Indicator (GMTI) sensors that provide high resolution and near-real time information for valuable moving targets such as battlefield vehicles. One important capability researched by recent studies [ref] is improved accuracy in the detection and classification of these vehicles. Battlefield vehicles are generally classified as tracked or wheeled vehicles based on the differences in their structure and movement patterns.

The movement of many targets can generally be decomposed into the global movement of the main body, and the local movement, also called micro-motion, of one of its components. For instance, the movement of a wheeled vehicle can be decomposed into the body part translation and the rotation of the wheels. The micro-motion of a moving target is unique and stable, therefore becomes a valuable cue to identify the target. Micro-motion produces modulation of radar echo of a moving target, and this phenomenon is called micro-Doppler effect. By analyzing the frequency of the modulated echoes, it has been found that significant differences between different targets exist, thus providing an indication that a micro-Doppler signature can be used as the basis for target classification or recognition (Chen et al., 2006).

Micro-Doppler based classification algorithms generally consist of the following stages: micro-Doppler signal separation, feature extraction, and classifier construction. The goal of micro-Doppler signal separation is to extract the relatively pure micro-Doppler signal from the clutter or the signal produced by the global movement, in different Radar mode. This step is essential for the subsequent feature extraction and classification stages.
Micro-Doppler based target classification applications have been employed for different target types, namely human activities (Kim and Hao, 2009), aerial targets (Molchanov et al., 2014), and ground targets (Li et al., 2011). Variant radar modes have also been used in the classification process including continuous wave radar (Kim and Hao, 2009) and SAR/GMTI (Li et al., 2011).

In general, a micro-Doppler signal is relatively weak and complicated, that normally brings significant challenges in its separation and as a result may lead to poor classification of targets. For example, in the case of wheeled/tracked vehicle classification, the micro-Doppler signal of the wheel or the track is mixed with the signals of the vehicle body and even those from the environmental clutter. The spectrum of the mixed signal from a moving vehicle is distributed in the range $\left[0,2 f_{d}\right]$, where $f_{d}$ is the Doppler frequency of the vehicle; the spectrum of the body signal is mainly distributed in the range $\left[0, f_{d}\right]$, while the spectrum of the micro-Doppler signal is distributed in the whole range (Li et al., 2011). More precisely, the micro-Doppler signal is generally non-linear and non-stationary (Smith, 2008), and as a result traditional signal separation methods such as frequency filtering may fail in this case. Therefore, various methods such as the wavelet transform (Thayaparan et al., 2007; Shah et al.,2015), chirplet transform (Thayaparan et al., 2005), Empirical Mode Decomposition (EMD) (Li et al., 2011), and discrete fractional Fourier transform (DFrFT) (Chen et al., 2014) have been applied for the separation of micro-Doppler signals. Both chirplet and DFrFT useadopt the fixed base functions requiring, there are a large number of parameters that need to be estimated during the decomposition. Comparinged withto the EMD decomposition, these transforms are more time-consuming. EMD, in particular, is an adaptive signal decomposition method, and has unique advantages in dealing with the nonstationary and non-linear signals and therefore has been receiving increasing attention. 
In this paper, two specific types of vehicles, i.e. the wheeled and the tracked, are classified according to their microDoppler signals in SAR/GMTI. In comparison to other micro-Doppler based classification tasks such as aerial target classification, battlefield vehicle classification brings additional challenges. These challenges can be summarised as follows: the ground clutter is stronger while the sky background is relatively clean; the radius and the velocity of wheels are both smaller than that of the helicopter rotor, thus the corresponding micro-Doppler signal is relatively weaker; potential occlusion of the wheel or the track by the vehicle body resulting in part of the micro-Doppler signal being unobservable; and this occlusion might be further influenced by the variant azimuth angle of SAR/GMTI sensors. In the latter case, there may be discontinuous signals or clutter in the echoes, resulting in uneven distribution of the extreme points. Thus, EMD would become ineffective due to the mode mixing problem (see, e.g. Tang et al., 2012; Hu and Hwang, 2012).

In this study, we overcome the mode mixing problem by applying Ensemble Empirical Mode Decomposition (EEMD) (Torres et al., 2011; Yeh et al., 2010) to extract the micro-Doppler signal. EEMD adds a white Gaussian noise signal, evenly distributed in whole frequency band, to the original signal to produce a new continuous signal. The latter is similar to the original signal and can be decomposed by EMD. The combined addition-decomposition operation is carried out several times, and the mean value of the multiple corresponding decomposition results is calculated as the final output of EEMD. By so doing, the interference of the clutter to the decomposition result is also diluted to a certain extent. In order to select some specific intrinsic mode functions (IMF), which are most correlative to the micro-Doppler signal, the correlation analysis is then carried out. The selected IMFs are used to extract four features based on their differences between the wheeled and the tracked vehicle signals. In the verification stage, we apply a support vector machine (SVM) classifier to the features extracted from the simulated data to categorise our test data as either wheeled or tracked vehicles.

The core contribution of this article is the EEMD based robust feature abstraction for the vehicle classification. This paper is organised as follows. Section 2 introduces the simulation of dual band SAR/GMTI radar echoes from wheeled and tracked vehicles. In Section 3 the details of the EEMD-based micro-Doppler signal separation procedure is presented. Section 4 outlines the vehicle classification method and verify its usefulness and capability. A conclusion and discussion is presented in the final section of the main text.

\section{Simulation of dual band SAR/GMTI radar echoes from wheeled and tracked vehicles}

In this paper, the radar echo including the micro-Doppler signal of the vehicles is generated by model simulation where the radar platform is the dual band SAR/GMTI. A scatter point model is chosen to represent and characterise the vehicles being tracked. Using this model, the global movement and local micro-movement of vehicles are represented by the different movement of the scatters. The radar echo is calculated in time-domain: the positions and the corresponding echo of each scatter are calculated respectively for each example point, and then these echoes are accumulated to produce the final simulation echo.

\subsection{Dual band SAR/GMTI model}

As shown in Fig. 1, the dual band SAR works with a single transmitting antenna $(D 0)$ and two receiving antennas $(D 1$ and $D 2$ ). The distance between the antennas is $B$.

Figure 1 Dual band SAR/GMTI

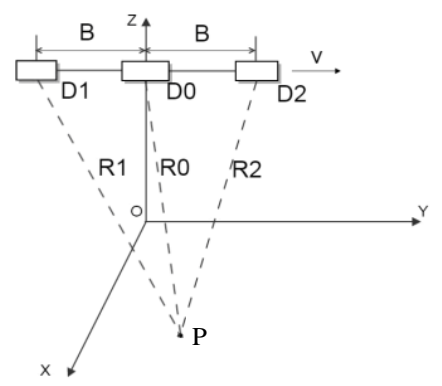

The signal transmitted from SAR is a series of chirp:

$$
\begin{aligned}
& s(t)=\sum_{n=-\infty}^{\infty} p(t-n \cdot P R T) \\
& p(t)=\operatorname{rect}\left(\frac{t}{T r}\right) e^{j \pi K_{r} t^{2}} e^{j 2 \pi f_{c} t}
\end{aligned}
$$

Here, $P R T$ is the pulse recurrence period, $T_{r}$ is the duration of the chirp signal, $\operatorname{rect}(\cdot)$ is a rectangular signal, $f_{c}$ is the carrier frequency of transmitted signal, $K_{r}$ is the chirp rate.

The echo signal is determined by the transmission signal waveform, antenna pattern, slant range, target RCS (Radar Cross Section), environmental and other factors. Without considering environmental factors, the single-point target radar echo signals can be written as:

$$
s_{r}(t)=\sum_{n=-\infty}^{\infty} \sigma \cdot w \cdot \mathrm{p}\left(t-n \cdot P R T-\tau_{n}\right)
$$

Here, $\sigma$ is the target point $\operatorname{RCS}, w$ is the antenna directional pattern bidirectional amplitude weighting, $\tau_{n}$ is the two-way time of electromagnetic wave between the radar and the target (Gierull, 2006):

$$
\begin{gathered}
\tau_{n}=\frac{2 R(s ; x, y, z)}{C} \\
R(s ; x, y, z)=\sqrt{\left(H-z_{s}\right)^{2}+x_{s}^{2}+\left(v \cdot s-y_{s}\right)^{2}}
\end{gathered}
$$

Here, $s$ is the slow time, $\left(x_{s}, y_{s}, z_{s}\right)$ is the corresponding three-dimensional position, $H$ and $v$ are the height and velocity of the carrier aircraft. 


\subsection{The scatter point model of vehicles}

The modelling and simulation of moving targets is carried out in the time domain. The basic principle is to calculate the target trajectory in its synthetic aperture time range according to its moving parameter: $\left\{\left(x_{s}, y_{s}, z_{s}\right) \mid s=t_{1}, t_{2}, \ldots t_{n}\right\}$. Thereafter, the echo signal in this time range is obtained according to the Formulae (2)(4).

\section{1) The wheeled vehicle}

The scattering point model is applied. Strong scattering points of the wheeled vehicle have two movements mode: the translation of the body part and the rotation of the wheel.

Figure 2 The wheel model

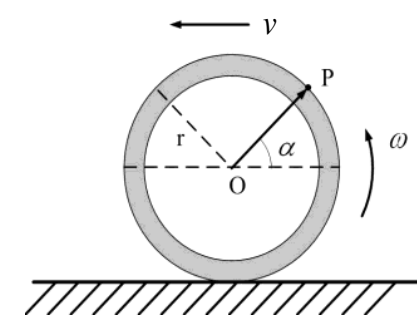

Suppose for a wheeled vehicle, the translation velocity is $v$ and the angular velocity of wheels is $\omega$.

The scatter point set $\left\{P^{v}, P^{w}\right\}$ is combined with two types of scatter points, where $P^{v}$ represents the global translation of the body part and $P^{w}$ represents the rotation of the wheel. Every scatter point has some important parameters such as the (RCS), movement type and velocity. The RCS of $P^{v}$ and $P^{w}$ are denoted by $\sigma^{v}$ and $\sigma^{w}$, respectively.

For the translation body part, the trajectory of the corresponding scattering point $P^{v}$ in the $\mathrm{X}-\mathrm{Y}-\mathrm{Z}$ axis (see Fig. 1) is given by:

$$
\left\{\begin{array}{c}
x_{s}^{v}=v \cdot s \cdot \sin (\theta) \\
y_{s}^{v}=v \cdot s \cdot \cos (\theta) \\
z_{s}^{v}=z_{0}
\end{array}\right.
$$

Here, $s$ is the slow time. Thereafter, the corresponding echo $s_{r}^{v}$ in this time range is obtained according to the Formulae (2-5).

For wheel rotation, the abstract model is constructed as in Fig. 2, and the trajectory of the corresponding scattering point in the $\mathrm{X}-\mathrm{Y}-\mathrm{Z}$ axis is:

$$
\left\{\begin{array}{c}
x_{s}^{w}=[r \cdot \cos (\omega \cdot s+\alpha)-r \cdot \cos (\alpha)+v \cdot s] \cdot \sin (\theta) \\
y_{s}^{w}=[r \cdot \cos (\omega \cdot s+\alpha)-r \cdot \cos (\alpha)+v \cdot s] \cdot \cos (\theta) \\
z_{s}^{v}=r \cdot \sin (\omega \cdot s+\alpha)-r \cdot \sin (\alpha)
\end{array}\right.
$$

Thereafter, the corresponding echo $s_{r}^{w}$ in this time range is obtained according to the Formulae $(2-4,6)$. Thus, the echo of wheeled vehicle is:

\section{2) The tracked vehicle}

$$
s_{r}^{v w}=s_{r}^{v}+s_{r}^{w}
$$

Consider a typical type of tracked vehicles, as shown in Fig. 3 , wherein the translational speed of the vehicle is $v$ and the wheel angular velocity is $\omega$.

Figure 3 The track model

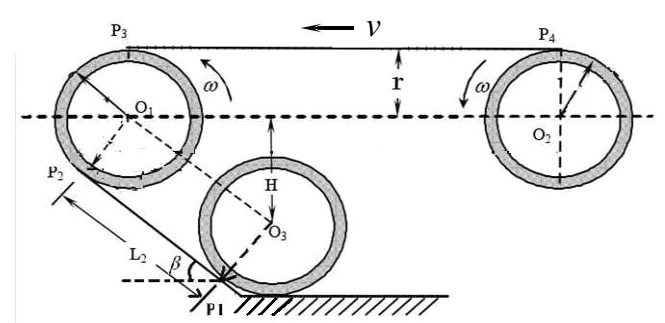

The corresponding scatter point set $\left\{P^{v}, P^{t}\right\}$ is combined with two types of scatter points, where $P^{v}$ represents the global translation of the body part and $P^{t}$ represents the complex movement of the track. Their RCS are $\sigma^{v}$ and $\sigma^{t}$, respectively.

For the translated body part, its trajectory is also calculated by Formula (5) and the corresponding echo $s_{r}^{v}$ is obtained according to the Formulae (2-5).

The movement of the tracked part is divided into three segments: a tilting movement (P1-P2), a rotation movement (P2-P3), and a translation movement (P3-P4). The trajectory of the corresponding scattering point in segment P1-P2 is:

$$
\left\{\begin{array}{c}
x_{s}^{t-p l}=[v \cdot s \cdot \cos (\beta)+v \cdot s] \cdot \sin (\theta) \\
y_{s}^{t-p l}=[v \cdot s \cdot \cos (\beta)+v \cdot s] \cdot \cos (\theta) \\
z_{s}^{t-p l}=-v \cdot s \cdot \sin (\beta)
\end{array}\right.
$$

The trajectory in segment P2-P3 is also calculated by Formula (5). The trajectory in segment P3-P4 is:

$$
\left\{\begin{array}{c}
x_{s}^{t-p 3}=2 \cdot v \cdot s \cdot \sin (\theta) \\
y_{s}^{t-p 3}=2 \cdot v \cdot s \cdot \cos (\theta) \\
z_{s}^{t-p 3}=z_{0}
\end{array}\right.
$$

The corresponding echo $s_{r}^{t}$ in this time range is obtained according to the Formulae (2-8). Thus, the echo of tracked vehicle is:

$$
s_{r}^{v t}=s_{r}^{v}+s_{r}^{t}
$$

Through the above models, the raw SAR/GMTI simulation signals of the main part movements and micro-movements are obtained in the range-azimuth plane. 


\section{EEMD-based micro-Doppler signal separation}

EEMD is applied to separate the micro-Doppler signal from the SAR/GMTI echo signal. Fig. 4 illustrates the flowchart for the separation procedure consisting of the three successive processing steps in the following order:

1) Pre-processing. In the raw SAR/GMTI echo, the microDoppler signal and the body part movement signal are distributed in the range-azimuth plane. Before the EEMD decomposition process, the raw echo signal of vehicles needs to be pre-processed to be a suitable input of EEMD.

2) EEMD decomposition. The pre-processed signal is thereafter decomposed by EEMD into several IMFs arranged in descending order of frequency and a residual.

3) Correlation analysis. This process is applied to evaluate the correlation between each IMF and the micro-Doppler and body part signal respectively. As a result, the IMFs which are most discriminative or correlative to microDoppler are further chosen.

\subsection{Pre-processing}

The main pre-processing steps include the range compression, range migration correlation, displaced phase centre antenna (DPCA) (Cerutti and Sikaneta, 2013) and projection. The micro-Doppler signal and the body part movement signal are focused in the range by the range compression and range migration correlation pre-processing (Chan and Koo, 2013). After that, DPCA is carried out to eliminate the static clutter signal and roughly localise the position of the moving vehicle. Finally, projection is applied to compress the signal from $2 \mathrm{D}$ to $1 \mathrm{D}$.

Figure 4 Flowchart of micro-Doppler signal extraction

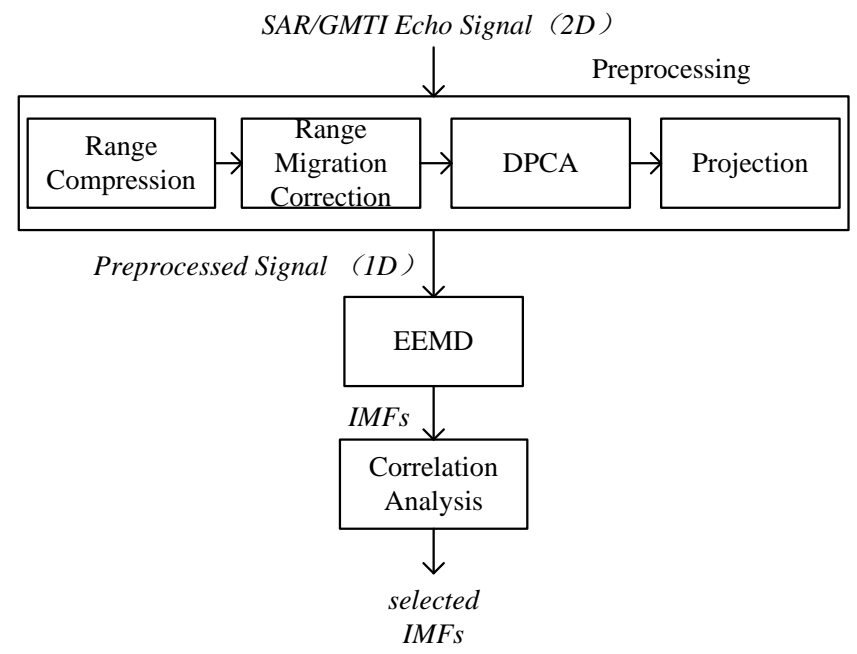

\subsection{EEMD decomposition}

EMD is an adaptive decomposition process and can decompose a signal into several IMFs arranged in frequency descending order and a residual, without the need to define any fixed basis functions in advance. EEMD, as an extension of EMD, performs better in the cases that the discontinuous signal, pulse interference signal or noise exists in the raw signal (Yeh et al., 2010).
Figure 5 Flowchart of EEMD algorithm

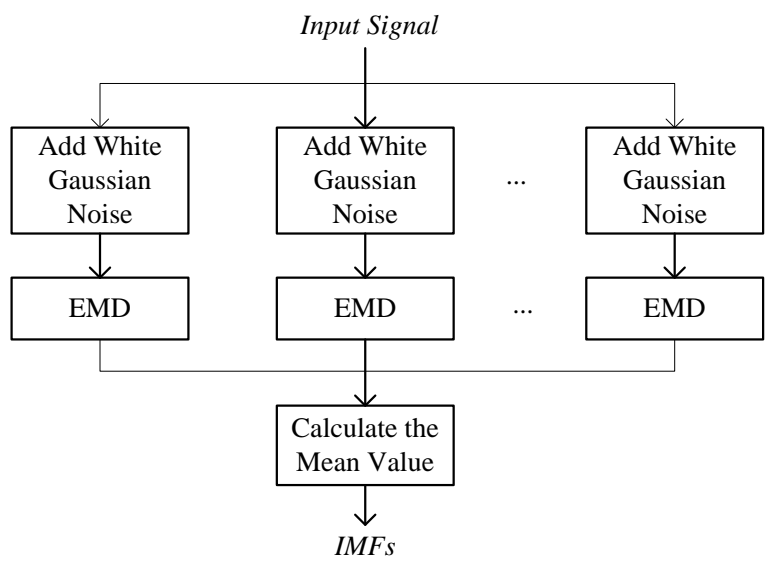

EEMD decomposition of the pre-processed signal is demonstrated in Fig.5. The key steps of EEMD decomposition are as follows:Step 1: Add white Gaussian noise to the original echo signal to make a new one.

Step 2: Obtain the set of IMFs and residual by EMD decomposition on the new signal:

$$
X(t)=\sum_{j=1}^{n} I M F_{j}^{e m d}+r^{e m d}
$$

$X(t)$ is the new signal, $I M F_{j}^{e m d}$ is the $\mathrm{j}$-th IMF and $r^{e m d}$ is the residual item.

Step 3: Repeat steps 1 and 2. The repetitions number is called the iteration number. The result for a given iteration $\mathrm{i}$ is:

$$
X_{i}(t)=\sum_{j=1}^{n} I M F_{i j}^{e m d}+r_{i}^{e m d}
$$

Here, $X_{i}(t), I M F_{i j}^{e m d}$ and $r_{i}^{e m d}$ represent the new signal, the $\mathrm{j}$-th IMF, and the residual item in the i-th iteration, respectively.

Step 4: Derive the final IMFs and residual, by calculating the average values of the IMFs and residuals in all iterations:

$$
\begin{aligned}
& I M F_{j}^{e e m d}=\sum_{i=1}^{N} I M F_{i j}^{e m d} / N \\
& r^{\text {eemd }}=\sum_{i=1}^{N} r_{i}^{\text {emd }} / N
\end{aligned}
$$

Here, $N$ is the iteration number.

In short, EEMD can be considered as a noise-assisted extension of EMD (Wu and Huang, 2011). The added white noise would uniformly distribute in the entire timefrequency space. When a signal is added to this uniformly distributed background, the bits of the signal at different scales are automatically projected onto proper scales. The result produced by each individual trial may be very noisy, however, since the noise in each trial is different, it would 
cancel out in the ensemble mean of a sufficient number of trials. The only persistent part is the signal as more and more trials are added in the ensemble.

\subsection{Correlation analysis}

The correlation analysis steps are as follows:

Step 1: Calculate the correlation coefficients between each IMF from the EMD/EEMD decomposition of the body part/ micro-Doppler signal:

$$
\lambda_{j}=\frac{\sum_{k}\left(x_{j k}-\bar{x}\right)\left(S_{k}-\bar{S}\right)}{\sqrt{\left(\sum_{k}\left(x_{j k}-\bar{x}\right)^{2}\right)\left(\sum_{k}\left(S_{k}-\bar{S}\right)^{2}\right)}}
$$

In Formula (12) $x_{j}$ is the $\mathrm{j}$-th IMF, $S$ is the body part signal or the micro-Doppler signal, $\lambda_{j}$ is the correlation coefficient between $x_{j}$ and $\mathrm{S}, x_{j k}$ is the $\mathrm{k}$-th element in $x_{j}, \bar{x}$ is the mean value of all the IMFs, and $\bar{S}$ is the mean value of $S$.

Step 2: Calculate the energy occupation ratios:

$$
\begin{aligned}
& \eta_{j}=\frac{E_{j}}{E_{\text {total }}} \\
& E_{\text {total }}=\sum_{j=1}^{n} E_{j}
\end{aligned}
$$

Here, $E_{j}$ is the energy of the jth IMF, $E_{\text {total }}$ is the sum of the energy value, and $\eta_{j}$ is the energy occupation rate of $\mathrm{j}$-th IMF.

Step 3. Filter out some trivial IMFs. There may be some IMFs with high correlation coefficient but low energy. These trivial IMFs with low energy occupation ratio could be removed.

\subsection{Experiments}

The micro-Doppler separation is carried out on the simulation echoes. Tab. 1 shows some key radar and target parameters in the simulation. The azimuth angle of radar is set in the range $\left(0^{\circ}-50^{\circ}\right)$, and the vehicle speed is set in the range $(0-20 \mathrm{~m} / \mathrm{s})$. In order to verify the effectiveness of EEMD decomposition, EMD is also employed to decompose both the 1D pre-processed signals of the wheeled and tracked vehicle.

Table 1 Main parameters of simulation experiment

\begin{tabular}{ll}
\hline Aircraft speed & $150 \mathrm{~m} / \mathrm{s}$ \\
Radar bandwidth & $150 \mathrm{MHz}$ \\
Aircraft altitude & $3000 \mathrm{~m}$ \\
Pulse repetition frequency & $750 \mathrm{~Hz}$ \\
Radar carrier frequency & $3 \mathrm{G} \mathrm{Hz}$ \\
Signal pulse width & $1.33 \mu \mathrm{s}$ \\
Target vehicle speed & $0-20 \mathrm{~m} / \mathrm{s}$ \\
Azimuth & $0^{\circ}-50^{\circ}$ \\
\hline
\end{tabular}

Figures 6 and 7 show the first four IMFs obtained by the EMD and EEMD decomposition and their spectrums, respectively. In Figs. 6 and 7, the spectrum of each IMF appears to overlap with the neighbouring IMF, particularly IMF1 and IMF2. This is likely due to a mode-mixing problem. However, the overlapping in Fig. 7 appears smaller than that in Fig. 6, which roughly confirmed that EEMD is better than EMD to overcome the mode-mixing problem. Further quantitative comparison and analysis would be illustrated in the subsequent correlation analysis.

Figure 6 EMD decomposition

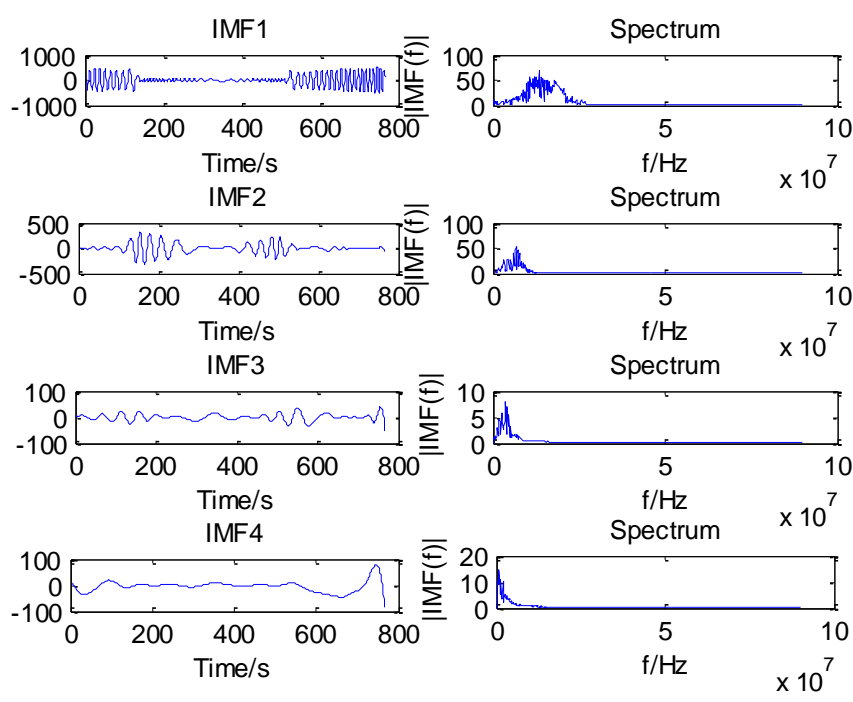

(a) EMD decomposition of wheeled vehicle

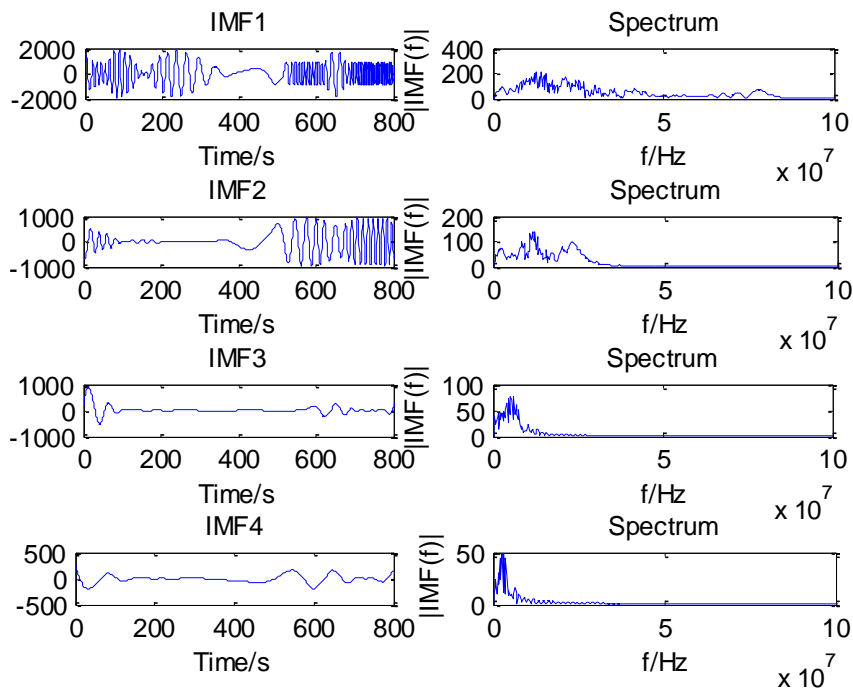

(b) EMD decomposition of tracked vehicles 
Figure 7 EEMD decomposition
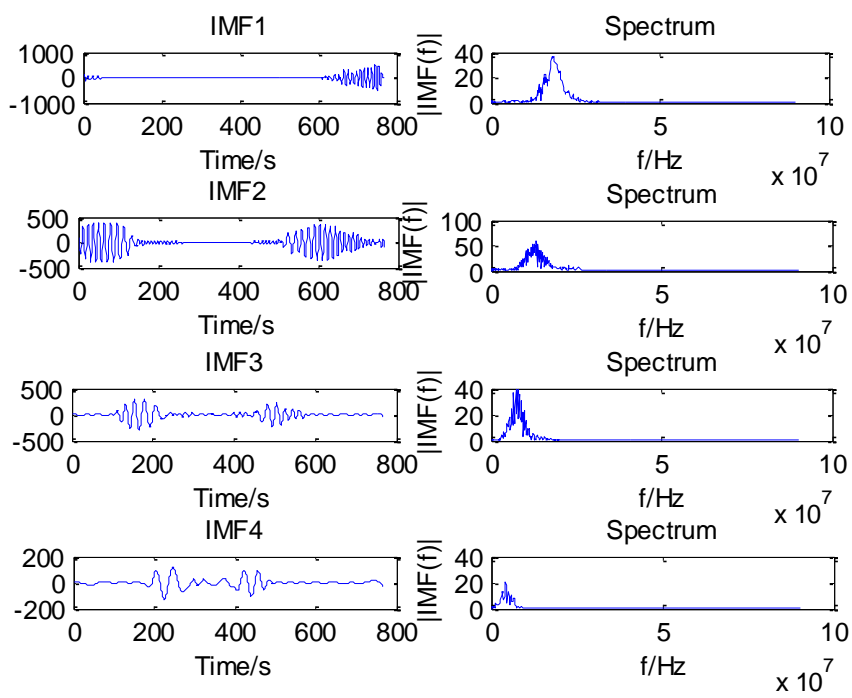

(a) EEMD decomposition of wheeled vehicle
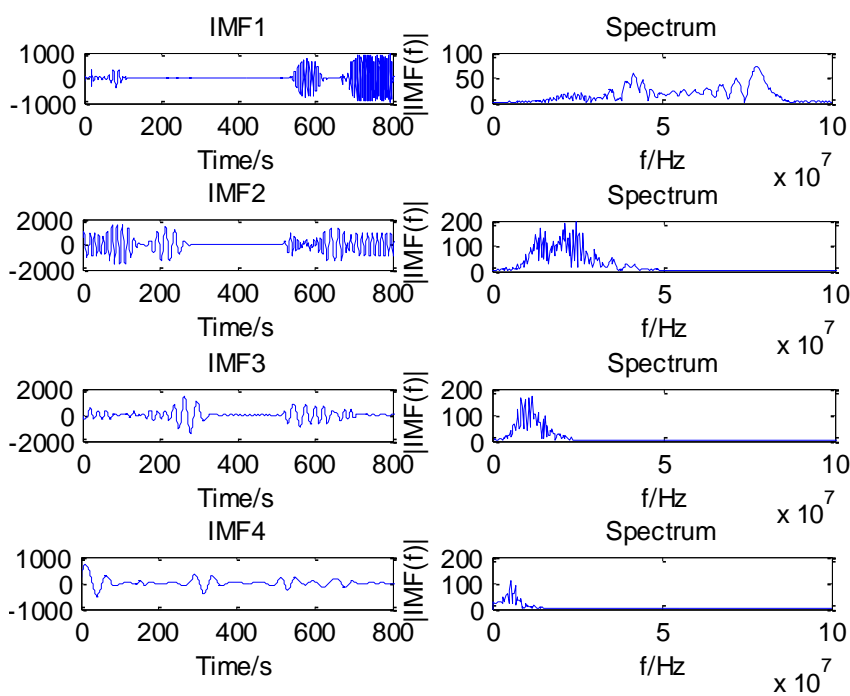

(b) EEMD decomposition of tracked vehicles

\subsubsection{Correlation analysis results}

The correlation coefficients between each IMF from EMD or EEMD decomposition and the body part or microDoppler signal are calculated respectively. Tables 2-8 show some important experimental results.

1) Effective IMF selection. In this experiment, IMFs with the correlation coefficient below a specific threshold are identified as uncorrelated. We observed that some IMF3 and IMF4 with the correlation coefficients higher than the selected threshold are filtered out due to their relatively low energy occupation ratio. This is illustrated in Table 2 that shows the correlation analysis of the first 7 IMFs for tracked vehicles. The correlation coefficients of IMF6 and IMF7 are lower than that of the first five IMFs. Overall, the first few
IMFs are highly useful. In the following experimental analysis, the correlation coefficient threshold is set 0.05 and only the first five IMFs are selected.

2) Effectiveness analysis of the EMD EEMD based microDoppler signal separation. Table 3 and Table 5 provide the IMFs generated for a wheeled vehicle. The tables show that IMFs from both the EMD and EEMD are almost uncorrelated with the micro-Doppler signal. However, for a tracked vehicle, we observed in Table 2 and Table 4 that the correlation coefficients between the IMFs from EEMD and the micro-Doppler signal are significantly higher than the corresponding part of EMD. The maximum and the mean correlation coefficient of the first 5 IMFs for EEMD are 0.22 and 0.09 respectively, whereas the corresponding numbers for EMD are 0.02 and 0.007. The micro-Doppler signal itself is a weak signal and is therefore sensitive to the interference of the mode-mixing problem. Thus, from our results, we observe that EEMD decomposition overcomes the mode-mixing problem more effectively leading to better performance in the micro-Doppler signal separation.

Table 2 EEMD decomposition correlation analysis of the tracked vehicle

\begin{tabular}{ccc}
\hline IMF & $\begin{array}{c}\text { Correlation } \\
\text { coefficient of body }\end{array}$ & $\begin{array}{c}\text { Correlation } \\
\text { coefficient of track }\end{array}$ \\
\hline IMF1 & 0.0105 & 0.2190 \\
IMF2 & 0.2207 & 0.3697 \\
IMF3 & 0.1169 & 0.2108 \\
IMF4 & 0.0719 & 0.2091 \\
IMF5 & 0.0402 & 0.0946 \\
IMF6 & 0.0196 & 0.0495 \\
IMF7 & 0.0066 & 0.0113 \\
\hline
\end{tabular}

Table 3 EEMD decomposition correlation analysis of the wheeled vehicle

\begin{tabular}{ccc}
\hline IMF & $\begin{array}{c}\text { Correlation } \\
\text { coefficient of body }\end{array}$ & $\begin{array}{c}\text { Correlation } \\
\text { coefficient of wheel }\end{array}$ \\
\hline IMF1 & 0.6778 & 0.00064 \\
IMF2 & 0.2365 & 0.0041 \\
IMF3 & 0.1500 & 0.0025 \\
IMF4 & 0.1310 & 0.0015 \\
IMF5 & 0.0532 & 0.0022 \\
\hline
\end{tabular}

3) IMF comparison between the wheeled and the tracked vehicle. As mentioned previously, the micro-Doppler signal of the wheeled vehicle is weaker than that of the tracked vehicle. As for the IMF, which can be seen as the combination of the body part signal component and the micro-Doppler signal component, the proportion of the 
micro-Doppler signal component against the body part signal component in the tracked vehicle is higher than that in the wheeled vehicle. Furthermore, even among the IMFs of the tracked vehicle, their proportions are different. Table 3 shows that the first two IMFs are highly correlated to the body part signal of the wheeled vehicle, but however, these IMFs have insignificant correlation with the micro-Doppler signal. Table 2 shows that the micro-Doppler signal is evenly distributed in the first four IMFs, and mainly, IMF1 contains the micro-Doppler signal component while IMF2 and IMF3 contain the body part signal components. These observations provide useful clues to select appropriate features for vehicle classification.

Table 4 EMD decomposition correlation analysis of the tracked vehicle

\begin{tabular}{ccc}
\hline IMF & $\begin{array}{c}\text { Correlation } \\
\text { coefficient of body }\end{array}$ & $\begin{array}{c}\text { Correlation } \\
\text { coefficient of track }\end{array}$ \\
\hline IMF1 & 0.0245 & 0.0157 \\
IMF2 & 0.0051 & 0.0007 \\
IMF3 & 0.0042 & 0.0009 \\
IMF4 & 0.0016 & 0.0006 \\
IMF5 & 0.0004 & 0.0003 \\
\hline
\end{tabular}

Table 5 EMD decomposition correlation analysis of the wheeled vehicle

\begin{tabular}{ccc}
\hline IMF & $\begin{array}{c}\text { Correlation } \\
\text { coefficient of body }\end{array}$ & $\begin{array}{c}\text { Correlation } \\
\text { coefficient of wheel }\end{array}$ \\
\hline IMF1 & 0.7755 & 0.02 \\
IMF2 & 0.0601 & 0.0009 \\
IMF3 & 0.0041 & 0.0017 \\
IMF4 & 0.0003 & 0.0034 \\
IMF5 & 0.0007 & 0.0016 \\
\hline
\end{tabular}

Table 6 EEMD correlation analysis of wheeled vehicles body part: azimuth angle $=10^{\circ}$

\begin{tabular}{ccc}
\hline & \multicolumn{2}{c}{ Speed } \\
\cline { 2 - 3 } IMF & $15 \mathrm{~m} / \mathrm{s}$ & $20 \mathrm{~m} / \mathrm{s}$ \\
\hline IMF1 & 0.3348 & 0.2331 \\
IMF2 & 0.2942 & 0.1175 \\
IMF3 & 0.1637 & 0.0980 \\
IMF4 & 0.0186 & 0.0101 \\
IMF5 & 0.0029 & 0.0034 \\
\hline
\end{tabular}

4) Robustness analysis of the EEMD decomposition against the key parameter variation. In the following experiments, we have varied two key parameters, azimuth angle and vehicle speed, during the decomposition process so as to observe the corresponding variation in the correlation coefficients. Tables 6-8 illustrate the results when a typical case for a wheeled vehicle is considered. Our results show that SAR target echo is sensitive to the azimuth variation with correlation coefficients of IMF2 remaining relatively stable for azimuth angle variations from $10^{\circ}$ to $45^{\circ}$. In general, the results of EEMD decomposition are fairly stable against the parameter variation.

Table 7 EEMD correlation analysis of wheeled vehicles body part: azimuth angle $=30^{\circ}$

\begin{tabular}{ccc}
\hline & \multicolumn{2}{c}{ Speed } \\
\cline { 2 - 3 } IMF & $15 \mathrm{~m} / \mathrm{s}$ & $20 \mathrm{~m} / \mathrm{s}$ \\
\hline IMF1 & 0.6778 & 0.3220 \\
IMF2 & 0.2365 & 0.2157 \\
IMF3 & 0.1500 & 0.1583 \\
IMF4 & 0.1310 & 0.0067 \\
IMF5 & 0.0532 & 0.0044 \\
\hline
\end{tabular}

Table 8 EEMD correlation analysis of wheeled vehicles body part: azimuth angle $=45^{\circ}$

\begin{tabular}{ccc}
\hline & \multicolumn{2}{c}{ Speed } \\
\cline { 2 - 3 } IMF & $15 \mathrm{~m} / \mathrm{s}$ & $20 \mathrm{~m} / \mathrm{s}$ \\
\hline IMF1 & 0.5757 & 0.7582 \\
IMF2 & 0.2843 & 0.1197 \\
IMF3 & 0.1336 & 0.0342 \\
IMF4 & 0.0190 & 0.0062 \\
IMF5 & 0.0061 & 0.0004 \\
\hline
\end{tabular}

\section{EEMD based vehicle classification}

Based on the EEMD micro-Doppler decomposition described in the previous sections, we present a vehicle classification approach that constitutes of two main stages: (1) feature extraction and (2) classifier training (refer to Fig.8). SVM (Chang and Lin, 2011; Zhang et.al., 2015; Zhu and Zhao, 2016) is applied for classification purpose.

\subsection{Micro-Doppler feature extraction}

Based on the above-mentioned differences in the microDoppler characteristics of the two types of vehicle considered, four new features are extracted for the classification of these two categories. These four features are as follows:

Feature 1: The energy in high frequency band of IMF1:

$$
W=\int_{f_{d}}^{2 f_{d}} x_{1}(f) d f
$$


Here, $f$, and $f_{d}$ are the frequency and target Doppler frequency, respectively. The high frequency band of IMF1 is integrated to realize the energy discrimination.

Feature2: The volatility in high frequency band of IMF1:

$$
F^{\prime}=\max \left(\left\{x_{1}(k)_{k=(t-1) u}^{t u}\right\}\right)-\min \left(\left\{x_{1}(k)_{k=(t-1) u}^{t u}\right\}\right)
$$

Here, $x_{1}$ is divided into $m$ parts with $t=(1,2,3 \ldots m)$, $u$ is the number of elements in each part, thus the total volatility degree is $F=\operatorname{sum}\left(F^{\prime}\right)$.

Figure 8 Flowchart of the vehicle classification algorithm

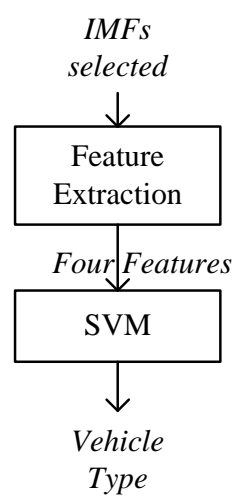

Features 1 and 2 describe the differences between the two types of vehicles in IMF1. The correlation analysis abovementioned show that IMF1 of the wheeled vehicle contains mainly the body part signal (shown in Tab.3, its correlation coefficient of body and wheel are 0.6778 and 0.00064 , respectively) with relatively narrow spectral range of $\left[0, f_{d}\right]$, while IMF1 of the tracked vehicle contains mainly the micro-Doppler signal. Since the reflection coefficient of tack part is large and the micro-motion is significant, resulting in the broadening of its spectral range; thus we observe that the energy and the volatility in the spectral range of $\left[f_{d}, 2 f_{d}\right]$ of the two types of vehicles are different.

Feature3: The maximum spectrum magnitude of IMF2:

$$
\max [Y]=\max \left(\left\{x_{2}(k)\right\}_{k=1}^{N}\right)
$$

Whereby, $Y$ is the magnitude of the Fourier transform of IMF2, $x_{2}(k)$ is the k-th element in the spectrum of IMF2.

Feature 3 reflects the differences between the two types of vehicles in IMF2. In general, the normalized energy of the body part signal is comparable between the wheeled and the tracked vehicles. The body part signal energy of the wheeled vehicle is mainly distributed in IMF1, while the body part signal energy of the tracked vehicle is mainly distributed in IMF2. The energy proportion in IMF2 of the tracked vehicle is relatively smaller than that of the wheeled vehicle. Therefore, the following conclusions can be drawn: the spectral magnitude of IMF2 of a wheeled vehicle is smaller than that of a tracked vehicle.
Feature 4: The dispersion among IMFs based on energy entropy:

$$
P=-\sum_{j=1}^{n} \eta_{j} \log \eta_{j}
$$

Here, $P$ is the dispersion between the various IMFs, $\eta_{j}$ is the energy percentage of the j-th IMF to the total energy.

Feature 4 is designed mainly according to the energy distribution of every IMF. The body part signal energy of the wheeled vehicle is mainly distributed in the first two IMF, while the micro-Doppler signal is fairly weak and almost negligible compared to the body part signal. The body part signal energy of the tracked vehicle is mainly distributed in IMF2 and IMF3, however the micro-Doppler signal is relatively strong due to the relatively big reflection coefficient of the track. In short, the energy distribution of the tracked vehicle is generally more even in each IMF than that of the wheeled vehicle, and therefore the corresponding dispersion degree may be smaller than that of the wheeled vehicle.

\subsection{Experimental results}

SVM is employed to classify the two types of vehicles considered (wheeled and tracked) on the basis of these features. Both the training and test sets comprise of simulated signals. The key radar parameters and target parameters in the simulation are provided in Table 6. The training and test examples are both produced randomly as per the parameter ranges shown in Table 6. The training sample set is further evenly divided into five subsets with each subset containing samples with the same intervals to ensure the consistency.

There are two main types of target in the test examples: wheeled vehicles and tracked vehicles. In order to test the target classification rate of the four features at variant parameters, the vehicle speed and the radar azimuth angle, 900 test samples are randomly generated within the four sub-range of vehicle speed and the four sub-range of radar azimuth angle. An SVM classifier is applied to different features in order to test the classification rates for variations in vehicle speed and radar azimuth angle respectively. The classification results are presented in Tables 9 and 10. The average classification rates under different vehicle speed and the radar azimuth angle are provided in Table 11.

It can be noted that the influence of varying the vehicle speed on the classification rate is similar to that of the radar azimuth angle (presented in Tables 9 and 10). The microDoppler features play a more significant role in the classification process, and thus, we can deduce that the stronger the micro-Doppler signal is, the higher the classification rate will be. Likewise, the higher the vehicle speed, the stronger the micro-motion observed. With a fixed vehicle speed, higher azimuth angles will result in larger the radial velocities, thus leading to stronger micro-Doppler signals. In Table 9, we observe that when the target vehicle speed increases, the classification rates of Feature 1 and 
Feature 2 increase simultaneously. The micro-Doppler signal generated from the vehicle running at low speed is relatively weak and distributes mainly in low frequency domain, therefore it is mainly represented in IMF2 and IMF3, while rarely in IMF1. As for the tracked vehicle, the energy and the volatility of IMF1 are not obvious.

Table 9 Classification rate of each feature at different speed

\begin{tabular}{cccccc} 
& \multicolumn{5}{c}{ Speed: $\mathrm{m} / \mathrm{s}$} \\
Feature Average \\
\cline { 2 - 5 } & $2-5$ & $6-10$ & $11-15$ & $16-20$ & \\
\hline 1 & $74.1 \%$ & $90.8 \%$ & $95.1 \%$ & $98.4 \%$ & $89.6 \%$ \\
2 & $54.0 \%$ & $78.8 \%$ & $93.5 \%$ & $96.5 \%$ & $80.7 \%$ \\
3 & $94.2 \%$ & $85.0 \%$ & $78.3 \%$ & $66.9 \%$ & $81.1 \%$ \\
4 & $98.5 \%$ & $73.0 \%$ & $61.1 \%$ & $57.6 \%$ & $72.6 \%$ \\
\hline
\end{tabular}

Table 10 Classification rate under different azimuth angles

\begin{tabular}{|c|c|c|c|c|c|}
\hline \multirow{2}{*}{ Feature } & \multicolumn{4}{|c|}{ Azimuth angle } & \multirow{2}{*}{ Average } \\
\hline & $0-10^{\circ}$ & $10-20^{\circ}$ & $20-30^{\circ}$ & $30-40^{\circ}$ & \\
\hline 1 & $79.4 \%$ & $91.1 \%$ & $92.2 \%$ & $95.2 \%$ & $90.8 \%$ \\
\hline 2 & $76.4 \%$ & $81.5 \%$ & $85.7 \%$ & $88.4 \%$ & $84.7 \%$ \\
\hline 3 & $98.2 \%$ & $89.4 \%$ & $71.5 \%$ & $67.8 \%$ & $78.8 \%$ \\
\hline 4 & $97.8 \%$ & $75.7 \%$ & $62.1 \%$ & $59.3 \%$ & $70.8 \%$ \\
\hline
\end{tabular}

Table 11 Average classification rate of each feature

\begin{tabular}{ccccc} 
& \multicolumn{5}{c}{ Feature } \\
\cline { 2 - 5 } & 1 & 2 & 3 & 4 \\
\hline Average & $90.2 \%$ & $82.7 \%$ & $79.9 \%$ & $71.7 \%$ \\
\hline
\end{tabular}

The classification trends of Feature 3 and Feature 4 are opposite to that of Feature 1 and Feature 2. Feature 3 and Feature 4 are more distinctive in low speed. With increasing speed, the frequencies of both the body part signal and micro-Doppler signal also increase, and thus, the distributions on IMFs of both signals may become more even (shown in Tables 6-8). As for Feature 3, the proportions of IMF2 in both the wheeled and the tracked vehicle decrease, the distinctiveness of Feature 3 also decreases. As for Feature 4, the dispersion degrees of the wheeled and the tracked vehicles both increase. However, the former may increase faster reducing the difference with the latter. In such a situation, the effectiveness of Feature 4 also decreases.

In short, the four features proposed are complementary during the classification process. In the case of lower speeds or smaller azimuth angles, the use of Feature 3 and Feature 4 results high classification rates. While, in the case of higher speeds or larger azimuth angles, Feature 1 and Feature 2 provide high classification rates. The experimental results presented in this paper show that the proposed method has a high classification rate even in complex environments or complex movements.

\section{Conclusion}

In this paper, the EEMD decomposition approach is applied for the detection and categorisation of wheeled and tracked vehicles under complex environments and complex motion modes. By adding Gaussian white noise, EEMD decomposition overcomes the model-mixing problem that has remained unresolved in literature. Correlation analysis is carried out to further verify the effectiveness of EEMD decomposition and for the selection of effective IMFs. Thereafter, four features are extracted based on differences of IMFs for the two types of vehicles. An SVM classifier is applied for the classification of tracked and wheeled vehicle signals. The experimental results presented show that classification algorithm is effective and the features are complementary for different target velocities and azimuth angles.

As for the extension capacity of this algorithm a more sophisticated classification algorithm could be constructed based on the moving target parameter estimation method (Gierull, 2006). In the improved approach the vehicle speed could be estimated from the vehicle body part signal to guide the feature selection process as mentioned in the analysis provided in the paper.

\section{Acknowledgements}

The first author wishes to thank Hangzhou Dianzi University, China to sponsor his recent six month research visit to the University of the West of England (UWE) Bristol, UK and also UWE for hosting his visit.

\section{References}

Cerutti, M.D. and Sikaneta, I. (2013) 'A generalization of DPCA processing for multi-channel SAR/GMTI radars', IEEE Transactions on Geoscience and Remote Sensing, Vol.51, no.1, pp.560-572.

Chan, Y.K. and Koo, V.C. (2008) 'An introduction to synthetic aperture radar (SAR)', Progress In Electromagnetics Research B, Vol.2, pp.27-60.

Chang, C. and Lin, C. (2011) 'LIBSVM: A library for support vector machines', ACM Transactions on Intelligent Systems and Technology, Vol. 2, Issue 3, Article No. 27.

Chen, V.C., Li, F., Ho, S.S. and Wechsler, H. (2006) 'Micro-Doppler effect in radar: phenomenon, model, and 
simulation study', IEEE Transactions on Aerospace and Electronic systems, Vol. 42, no. 1, pp.2-21.

Chen, X., Guan, J., Bao, Z. and He, Y. (2014) 'Detection and extraction of target with micromotion in spiky sea clutter via short-time fractional Fourier transform', IEEE Transactions on Geoscience and Remote Sensing, Vol.52, no.2, pp.1002-1018.

Gierull, C.H. (2006) 'Ground moving target parameter estimation for two-channel SAR', IEE Proceedings Radar Sonar and Navigation, Vol.153, no.3, pp.224-233.

$\mathrm{Hu}$, X., Peng, S. and Hwang, W.L. (2012) 'EMD revisited: A new understanding of the envelope and resolving the mode-mixing problem in AM-FM signals', IEEE Transactions on Signal Processing, Vol.60, no.3, pp.10751086.

Kim, Y. and Hao, L. (2009) 'Human activity classification based on micro-Doppler signatures using a support vector machine', IEEE Transactions on Geoscience and Remote Sensing, Vol. 47, no. 5, pp.1328-1337.

Li, Y., Du, L. and Liu, H. (2011) 'Moving vehicle classification based on micro-Doppler signature', In IEEE International Conference on Signal Processing, Communications and Computing (ICSPCC), pp.1-4, INSPEC Accession Number: 12330573, DOI: 10.1109/ICSPCC.2011.6061714.

Molchanov, P., Egiazarian, K., Astola, J., Totsky, A., Leshchenko, S. and Jarabo-Amores, M.P. (2014) 'Classification of aircraft using micro-Doppler bicoherencebased features', IEEE Transactions on Aerospace and Electronic systems, Vol.50, no. 2, pp.1455-1467.

Smith, G.E. 'Radar target micro-Doppler signature classification', $\mathrm{PhD}$ thesis, Department of Electronic and Electrical Engineering, University College London, 2008.

Tang, B., Dong, S. and Song, T. (2012) 'Method for eliminating mode mixing of empirical mode decomposition based on the revised blind source separation', Signal Processing, Vol.92, no.1, pp.248-258.

Thayaparan, T., Abrol, S. and Qian, S. (2005) 'MicroDoppler analysis of rotating target in SAR', Defence R\&D Canada Ottawa, Technical Memorandum, DRDC Ottawa TM 2005-204.

Thayaparan, T., Abrol, S., Riseborough, E., Stankovic, L.J., Lamothe, D. and Duff, G. (2007) 'Analysis of radar microDoppler signatures from experimental helicopter and human data', IET Radar, Sonar \& Navigation, Vol.1, no.4, pp. 289299.

Torres, M.E., Colominas, M.A., Schlotthauer, G. and Flandrin, P. (2011) 'A complete ensemble empirical mode decomposition with adaptive noise', IEEE International Conference on Acoustics, Speech and Signal Processing (ICASSP), pp. 4144-4147. INSPEC Accession Number: 12176054, DOI: 10.1109/ICASSP.2011.5947265.
Wu, Z.H and Huang, N.E. (2011) 'Ensemble empirical mode decomposition: a noise-assisted data analysis method.' Advances in Adaptive Data Analysis, Vol.1, no. 1, pp.1-41.

Yeh, J.R., Shieh, J.S. and Huang, N.E. (2010) 'Complementary ensemble empirical mode decomposition: A novel noise enhanced data analysis method', Advances in Adaptive Data Analysis, Vol.2, no.2, pp.135-156.

Zhu, C. and Zhao, H. (2016) 'Least square support vector machine for structural reliability analysis', International Journal of Computer Applications in Technology, Vol.53, no.1, pp.51-61. 